\title{
Practical solutions for nonlinear accelerator lattice with stable nearly regular motion
}

\author{
V. Danilov \\ Spallation Neutron Source Project, Oak Ridge National Laboratory, Building 8600, Oak Ridge, Tennessee 37830, USA
}

(Received 21 August 2008; published 20 November 2008)

\begin{abstract}
The use of nonlinear lattices with the large betatron tune spreads can increase instability and space charge thresholds by orders of magnitude compared to typical linear accelerator lattices. Unfortunately, strong nonlinear fields create, in general, strong resonances and chaotic motion. This shrinks the dynamic aperture to impractical values, thus erasing all benefits from their use. Previously known examples of stable and regular accelerator motion with special nonlinear lenses were related to one-dimensional motion or round beams. However, no solution has been realized with real 2D transverse magnetic fields to produce stable, close to regular 2D motion with the large dynamic aperture and betatron tune spread comparable to the betatron tune itself. This paper presents possible solutions for such $2 \mathrm{D}$ lattices. They consist of straight sections with short linear and nonlinear lenses with transverse magnetic fields.
\end{abstract}

DOI: 10.1103/PhysRevSTAB.11.114001

PACS numbers: 05.45. $-\mathrm{a}, 45.50 .-\mathrm{j}$

\section{INTRODUCTION}

Accelerator lattices with regular nonlinear motion, having analytical invariants in coordinates and momenta, represent an obvious generalization of linear lattices with Courant-Snyder invariants. Unfortunately, thin, or at least not continuous, nonlinear lenses (sextupoles, octupoles, etc.), placed into a linear lattice, produce nonlinear resonances with extremely complicated chaotic motion in their vicinity, and unstable motion with unlimited amplitude growth. The invariants of motion in these cases are not analytic functions of coordinates and momenta-this is the reason for the complex behavior. However, there exist some special combinations of multipoles, either thin or distributed, for which the invariants become analytic functions of dynamic variables. For these ideal unperturbed systems, the resonances become either isolated in the phase space or disappear completely - the latter 1D case is presented in the next section. The time dependence of the coordinates (at least in the particular $1 \mathrm{D}$ case) can be obtained by integrating some function of the coordinates and finding its inverse function similar to the 1D time independent case. That is why these systems are called integrable. For the simplest 1D integrable system, its invariant, in some sense, replaces the Hamiltonian of the 1D time independent system in the sense that its constant levels show the invariant lines in the phase space.

The development of lattices with invariants of motion started in application to round colliding beams [1]. The idea was to reduce the transverse motion to a 1D case, thus eliminating harmful 2D resonances. A few years later, many examples of 1D integrable lattices were found [2]. Some had been discovered earlier by McMillan et al. [3]. Independently, numerical methods to eliminate resonances and achieve regular motion were suggested in [4] and further developed in [5-7]. Some of these systems were extended to the $2 \mathrm{D}$ case of round beams [8] and to trans- verse lenses formed by electron beams [9]. Also, exactly integrable 2D cases with magnetic field lenses were found [8], but all of these yielded unstable transverse motion. A previous attempt to find exact 2D stable cases with magnetic lenses failed (some attempts to investigate the 2D case can be found in [10]). The main problem with real magnetic fields is that the horizontal and vertical components are linked to each other through Maxwell equations. For example, linear lenses focus particles in one plane and defocus in the other. In nonlinear cases the motion in the horizontal and vertical planes is inevitably coupled, reducing degrees of freedom for the fields to make the motion fully integrable. In this paper we exploit capabilities of linear lattices to squeeze the beam in one plane at a nonlinear lens, which then influences mostly the other plane. In accelerator language, we create a very large beta function beating and place the nonlinear lenses where the ratio of horizontal and vertical beta functions is large. These lenses affect mostly one degree of freedom, that in which the beta function is large. Furthermore, if this nonlinear kick resembles the lens for an integrable 1D case, the overall 2D motion becomes almost decoupled and nearly integrable. Here I present the simplest 2D accelerator lattices with large beta function beating and the necessary conditions to create very nonlinear nearly integrable stable $2 \mathrm{D}$ motion.

\section{UNDERLYING 1D LATTICE}

To begin, consider a simple lattice having one straight section with the length $L$ and one infinitely thin lens. We always can transform the variables $x \rightarrow x \sqrt{L}, x^{\prime} \rightarrow p / \sqrt{L}$ so that we can work with length $L \equiv 1$ for the map in the new variables. The corresponding map for the new coordinate $x$ and momentum $p$ is

$$
\bar{x}=x+p, \quad \bar{p}=p-\frac{2 a \bar{x}^{3}+3 b \bar{x}^{2}+c \bar{x}+d}{a \bar{x}^{2}+b \bar{x}+e},
$$


where the bar sign denotes the new variables, and $a, b, c, d$, $e$ are arbitrary parameters. One can check that the following function is invariant under this transformation:

$$
\begin{aligned}
I\left(x, x^{\prime}\right)= & \left(a x^{2}+b x+e\right) p^{2}+\left(2 a x^{3}+3 b x^{2}+c x+d\right) p \\
& +a x^{4}+2 b x^{3}+c x^{2}+2 d x .
\end{aligned}
$$

The details on how to find this and other integrable lattices can be found in [5]. The nonlinear kick in (1) can be represented in more convenient form as follows:

$$
\begin{aligned}
-\frac{2 a x^{3}+3 b x^{2}+c x+d}{a x^{2}+b x+e}= & -2 x \\
& -\frac{b x^{2}+(c-2 e) x+d}{a x^{2}+b x+e} .
\end{aligned}
$$

The linear part of the force with a straight section map gives a linear phase advance of $\pi / 2$ - this is the frequency of the motion for the large values of the invariant (2).

The choice of linear transformation between nonlinear kicks does not necessarily have to be a straight section and a linear focusing kick $-2 x$. Since the nonlinear kick parameters in the right-hand side (RHS) of (3) are arbitrary, we can always change the scale of coordinate and add a linear function of coordinate to momentum (in other words, we can make a linear canonical transformation that is equivalent to the change of beta function and its derivative at the lens) to change the linear matrix between the lenses to produce another integrable map. But one property of the new system remains the same-it will always have the same eigenvalues (or frequencies). Therefore, any linear map with eigenfrequency $\pi / 2$ and nonlinear kick of the form of the second term in the RHS of (3) constitutes an integrable system. Its invariant is always given by (2) but with an appropriate change of variables, determined by the choice of the linear matrix. In our case of straight section with $L=1$ and thin kick given by (3), the invariant is exactly given by (2). It is easy to check that the phase advance $-\pi / 2$ will work as well. In general, quadratic invariants of the type (2) exist for any lattice with fractional tunes $\pm 1 / 4$ and kicks

$$
-\frac{g_{1} x^{2}+g_{2} x+g_{3}}{g_{4} x^{2}+g_{1} x+g_{5}}
$$

where the $g_{i}$ are arbitrary parameters. It is essential to observe that the nonlinear lens has a quadrupole component and linear lattice tunes $\pm 1 / 4$ are not simply the betatron tune motion near the closed orbit. Because the quadrupole term in (4) is one of arbitrary parameters, we do not have any constraints on the betatron tunes.

In the next sections we present 2D cases, based on this 1D map. It is shown that it is possible to find such 2D lattices when the horizontal and vertical motions are very much decoupled and each plane has invariants of the form (2).

\section{2D THIN NONLINEAR LENSES}

Transverse magnetic focusing in accelerators can be achieved by solenoids with longitudinal fields and lenses with the transverse fields. The implementation of nonlinear kick of the form (3) using solenoids was suggested in [11]. Unfortunately, solenoids provide small focusing at large energies. In addition, the system is integrable for round beams, which requires equal tunes and equal beta functions at the nonlinear kick points. In contrast to this example, in the present paper we deal with transverse fields and thin lens kicks only. The term "thin lens" means that the length of the element is much shorter than the beta functions in each plane. At the same time we assume that this length is much longer than the beam aperture so that fringe fields of all elements can be neglected. The horizontal and vertical fields obey Maxwell's equations and are coupled to each other. The simplest representation of the kick can be given in complex variables $z=x+i y$ and the momentum transformation through the nonlinear lens has the form

$$
p_{x}=\operatorname{Re} F(z), \quad p_{y}=-\operatorname{Im} F(z),
$$

where $F(z)$ is any analytic function. The map (4) in this form is automatically symplectic and the fields $H_{x}$ (producing kicks in the $y$ direction) and $H_{y}$ (producing kicks in the $x$ direction) obey Maxwell's equation.

\section{CONSTRUCTING A SUITABLE LINEAR LATTICE}

As was pointed out in Sec. II, any 1D lattice with a $\pm \pi / 2$ phase advance and nonlinear kick (4) produces integrable motion with a quadratic invariant in momentum. Our goal is to construct a 2D lattice with nearly decoupled $x-y$ motion having an invariant of the type (2). If we have enough lenses and straight sections, we can then, in principle, produce a lattice having two places with very large beta function ratios, one with large $\beta_{x} / \beta_{y}$ and the other with large $\beta_{y} / \beta_{x}$. The special kicks then mostly influence only one degree of freedom at each location. It is also easy enough to make linear phase advances equal to $\pm \pi / 2$. Here we investigate the simplest lattices with suitable conditions.

Doublet case.-The simplest 2D linear lattice is a doublet. Each plane has a focusing and a defocusing lens. For simplicity, we would like to have horizontal and vertical degrees of freedom similar to each other, so the simplest doublet should have the same absolute value of quadrupole focusing and the same length $L$ of the two straight sections. This lattice does not meet our purpose for the following reason: the betatron phase advance condition $\left(\varphi_{b}=\right.$ $\pm \pi / 2$ ) can be satisfied only for one value of the integrated quadrupole gradients. The resulting matrix of the doublet at the beginning of straight section (with focusing lens at the end) is 


$$
M=\left|\begin{array}{cc}
1+\frac{L}{F} & 2 L+\frac{L^{2}}{F} \\
-\frac{L}{F^{2}} & 1-\frac{L}{F}-\frac{L^{2}}{F^{2}}
\end{array}\right|,
$$

where $F$ is the focal length of the focusing quadrupole, and $-F$ is the defocusing strength of the other quadrupole. To obtain the $\pm \pi / 2$ phase advance, the trace of this matrix must be zero. The only solution is $F=L / \sqrt{2}$. The maximum ratio of horizontal and vertical beta functions in this case is achieved at the point of the focusing (horizontally) quadrupole and is $\frac{4+\sqrt{2}}{4-\sqrt{2}} \approx 2.1$. This ratio is too small to decouple the horizontal and vertical motion when the nonlinear kick is included (as is shown in the next section, really good decoupled nearly integrable motion occurs when this ratio is greater than 50).

Triplet case.-This case is not symmetric between the horizontal and vertical directions, even though the matrices for $x$ and $y$ motion can be equalized. If all straight sections are the same length and the first and the third quadrupole strengths are identical, this case has two free parameters, namely, the ratios of the straight section length and the focal lengths of the central and the first (and the third) quadrupoles, respectively. Similar to doublet analysis, the solutions can be easily obtained and analyzed. The maximum ratio of beta functions can be as large as 6.9 in one plane and 2.4 in the other plane, where the central quadrupole focuses the particles. There still is not enough freedom to constrain the phase advances and obtain sufficiently large beta function ratios.

Quadruplet case.-For the symmetric quadruplet we have two choices: two doublets with alternating gradients of adjacent quadrupoles, or two focusing followed by two defocusing quadrupoles. The latter provides larger beta function ratios, and is therefore our choice for the linear lattice. This is the first symmetric lattice that has sufficient freedom to allow arbitrarily large beta function ratios at the nonlinear lens position, and phase advances of $\pm \pi / 2$ in both planes. Thus, if we obtain a solution for one plane, the other plane is automatically satisfied. To apply this principle we assume a symmetric quadruplet with two identical focusing quadrupoles and two identical defocusing quadrupoles having the same absolute value of the gradient. The straight sections have two different lengths: the shorter sections surrounded by longer ones, and vice versa. The symmetry gives the following simplifications-the tunes for horizontal and vertical motions are automatically the same and the horizontal beta functions produce the vertical beta function under mirror transformation around the center of straight section between focusing and defocusing quadrupoles (we later call it S1). Therefore, once the large ratio of beta functions is obtained in one plane, there is a symmetry point with the same conditions in the other plane.

The resulting lattice has the following sequence in the horizontal direction F-S0-F-S1-D-S0-D-S1, where F, D stand for focusing and defocusing quadrupoles with the same absolute value of the gradient, S0 is the base straight section with length unity (for simplicity, every focal and all other lengths will be specified in units of this length), and $\mathrm{S} 1$ is a variable length straight section. Thus, the lattice has two free parameters - the absolute value of the quadrupole gradient, and the length of the variable straight section. Once we specify the frequency (the only accessible good solution for us in this lattice has tunes of 0.75 in both planes), we are left with only one parameter. The choice of this parameter is up to us and we select the variable straight section length. The ratio of beta functions is a function of this parameter in our setup. We have two good choices for the location of the horizontal nonlinear kick - the focusing quadrupole position (1st choice) and the center of the straight section between the defocusing quadrupoles (2nd choice). In both cases the ratio of horizontal and vertical beta function can be made as large as desired. Figure 1 shows the dependence of these ratios on the length of the variable section $p$ (the tunes for the linear part lattice are 0.75 here for both planes). One can see that the larger ratio is achieved at the center of the section between two defocusing quadrupoles. But this location has one serious disadvantage: even though the ratio of beta functions is large, the horizontal beta function itself is rather small (of order unity in units of S0 length), as compared to the beta function at the focusing quadrupoles (which is of order 100). Therefore the nonlinear lens has to be much stronger for the second choice. In the next section we use the 1st choice and show the nonlinear motion for various beta function ratios and various initial conditions.

The behavior of beta functions is shown in Fig. 2 for $p=2$. The starting point of the plot is the first horizontally focusing quadrupole. The nonlinear lens for the horizontal

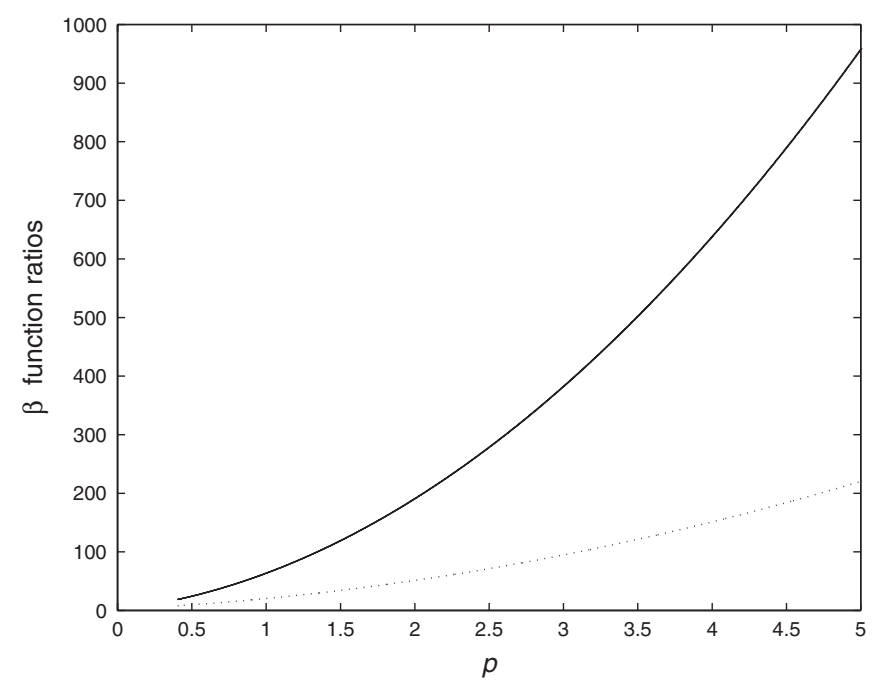

FIG. 1. The horizontal and vertical beta function ratios at the focusing quadrupole (dashed line) and the center of the straight section in between of the defocusing quadrupoles (solid line) as a function of the variable straight section length $p$. 


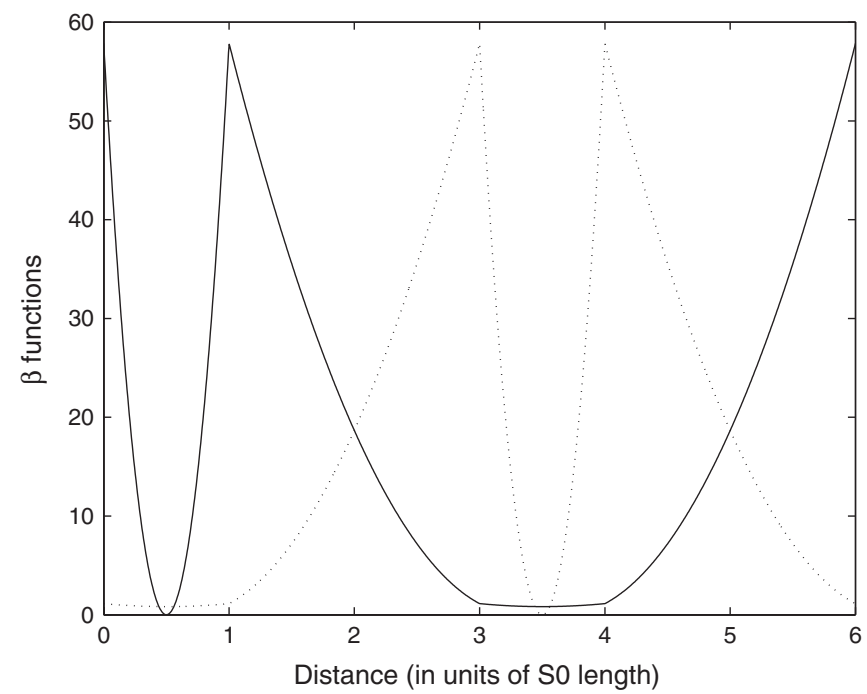

FIG. 2. Horizontal (solid) and vertical (dashed line) beta functions for $p=2$.

tune spread is located at the very beginning of the lattice; the vertical nonlinear lens is located at its center.

\section{NONLINEAR DYNAMICS FOR VARIOUS BETA RATIOS}

In this section we explore the quadruplet linear lattice and two nonlinear kicks that are placed at the focusing (horizontal kick) and defocusing (vertical kick) lenses such that the nonlinear lenses are separated by two straight sections. The kicks are intended to produce the nonlinear motion for both directions and they have the same parameters for their respective planes. Therefore, there is absolute symmetry for the horizontal and vertical planes. All the coordinate parameters are measured in terms of the S0 straight section length. The kick has the form (5) with $p_{x}=$ $\operatorname{Re} \frac{a z}{b z^{2}+1}, p_{y}=-\operatorname{Im} \frac{a z}{b z^{2}+1}$, where $z=x+i y$, and parameters $a, b$ are real for the horizontal nonlinear lens, and $p_{y}=$ $\operatorname{Re} \frac{a z}{b z^{2}+1}, p_{x}=-\operatorname{Im} \frac{a z}{b z^{2}+1}$, with the same parameters and notations for the vertical plane (note the swap of subscripts for $p$ from the horizontal to the vertical lenses). Figure 3 presents the exactly integrable $1 \mathrm{D}$ case when the initial $y$, $p_{y}$ are equal to zero and $a=-0.03 / \beta_{\max }$ (only for the lens with large $x$ beta function that we call $\beta_{\max }$; the other lens strength is equal to zero), $b=1$ for the lattice with the $\mathrm{S} 1$ length equal to 0.5 . The horizontal and vertical coordinates in this and all other figures of this section correspond to normalized variables; for normalization we use linear lattice Twiss parameters, taken at the beginning of straight section S1. The figure shows cases for ten various initial conditions, corresponding to ten different invariant lines; each line consists of 1000 points.

To find the invariant in this case we express variables of Sec. I via present coordinates such that the constructed lattice matrix takes the form (1) with linear kick from RHS

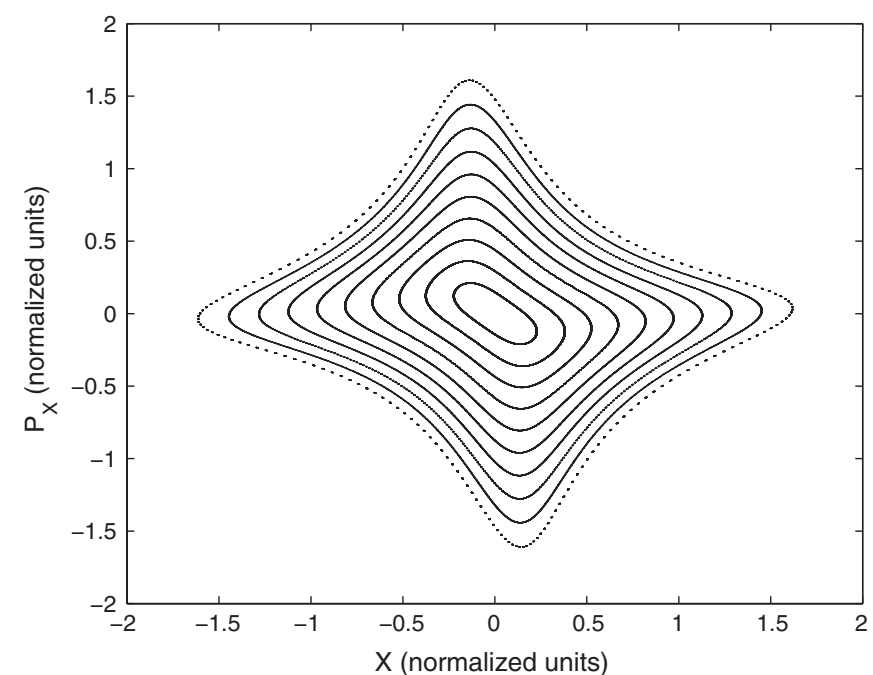

FIG. 3. 1D phase space.

(3) (as we mentioned, it is possible to do only if the matrices have the same eigenvalues, and this condition was met in course of the lattice construction). After expressing the old variables in terms of the present coordinate and momentum, one has to substitute these into (2) to get the invariant in the real lattice variables [one has to remember that if the fractional tune is -0.25 the opposite sign straight section length and linear kick $2 x$ are taken in (1) and (3)].

For the $2 \mathrm{D}$ case investigation, we use the same initial amplitudes for horizontal and vertical planes with $a=$ $-0.03 / \beta_{\max }, b=58 / \beta_{\max }$ for both lenses, where $\beta_{\max }$ is the largest of the beta functions at the lens-the division of the lens coefficients by the beta function is done to have the same tune shift and tune spread from nonlinear lens for different beta functions. Figure 4 shows three cases for approximately the same ten initial conditions for all plots but for various ratios of horizontal and vertical beta functions (we call it $B$ below) at the nonlinear kicks. One can see that, for low values of $B$, the motion is coupled and chaotic at large amplitudes. When $B$ approaches 50, the motion in both planes starts to look like decoupled 1D motion in the large dynamic range of coordinates and momenta. For values of $B$ above 100, the motion fully resembles that of the $1 \mathrm{D}$ case, and the dynamic aperture is almost infinite.

For all these cases, the linear lattice tune is 0.75 and the spread of frequencies is around 0.2. It means that, roughly at every unit of betatron frequency we can achieve around $30 \%$ of the tune spread with regular nonlinear motion. Here we introduce a simplified criterion for the lattice-it is good for producing nonlinear spread if the motion is still nearly regular and stable for amplitudes having $90 \%$ of the maximal tune shift. We concluded that it happens for values of $B$ above 50 that correspond to the center plots of the Fig. 4. 

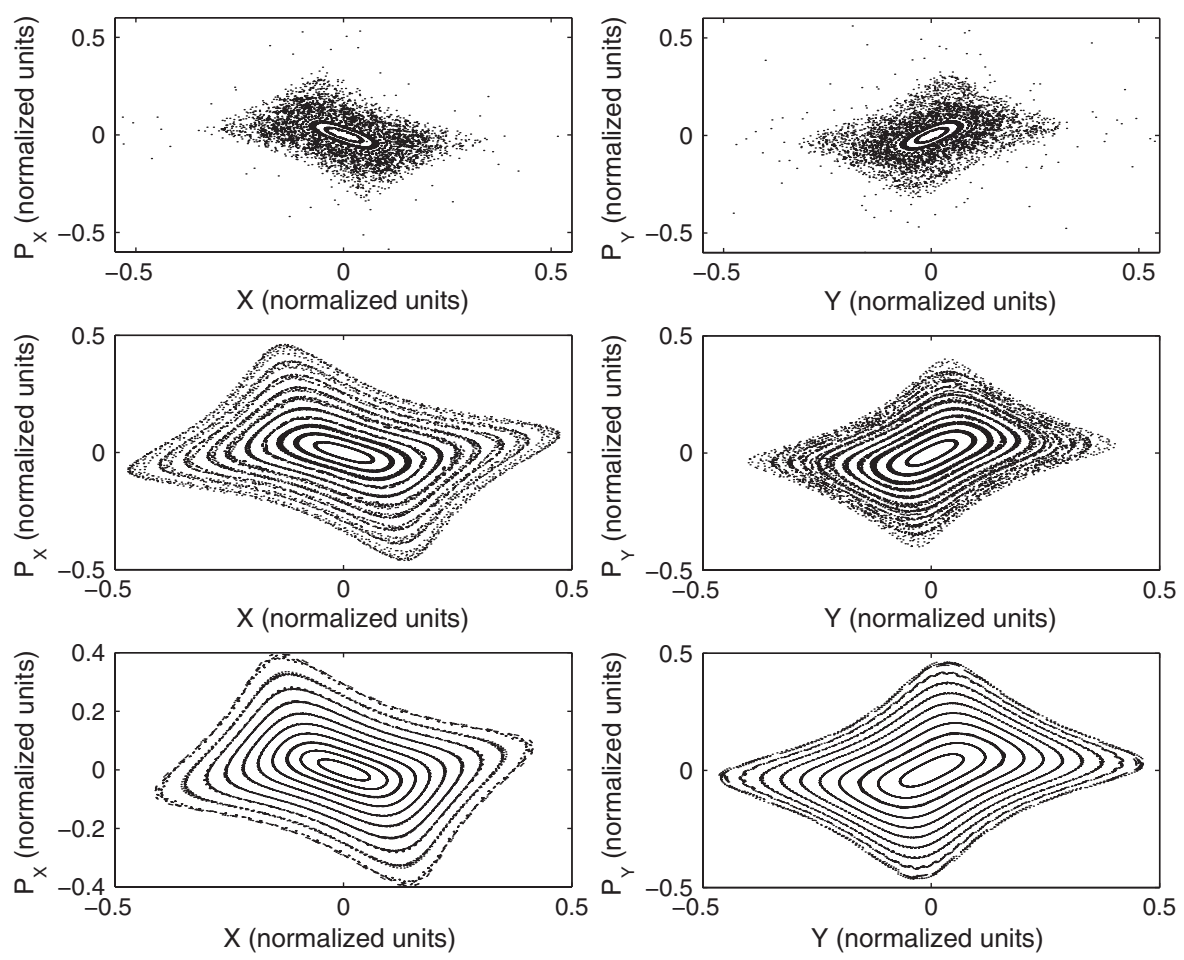

FIG. 4. Horizontal (left) and vertical (right) phase spaces for 3 different beta ratios $B: B \approx 10$ (top), $B \approx 58$ (middle), $B \approx 260$ (bottom).

Figure 5 shows what happens when one uses only an octupole component instead of an "integrable" lens. We took the second case, shown in Fig. 4, and used exactly the same linear part and octupole term as the nonlinear lens, nullifying all other terms. The available phase space shrinks a factor of 5 in each dimension (note that the scale in Fig. 5 is 5 times smaller than that in Fig. 4). In addition, the motion becomes very chaotic at large amplitudes with
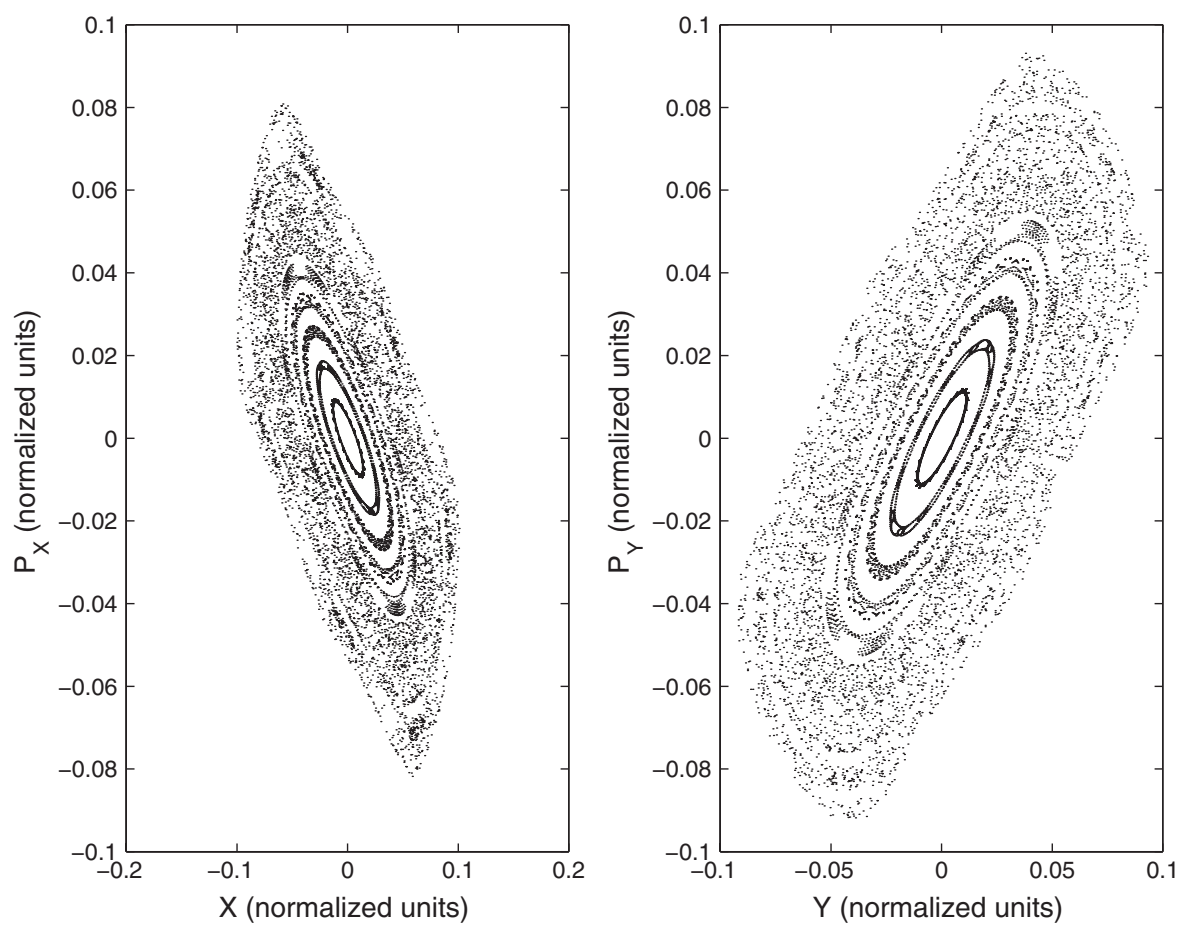

FIG. 5. Octupole phase space for the horizontal (left) and vertical (right) motion for the beta ratios equal to 58 . 


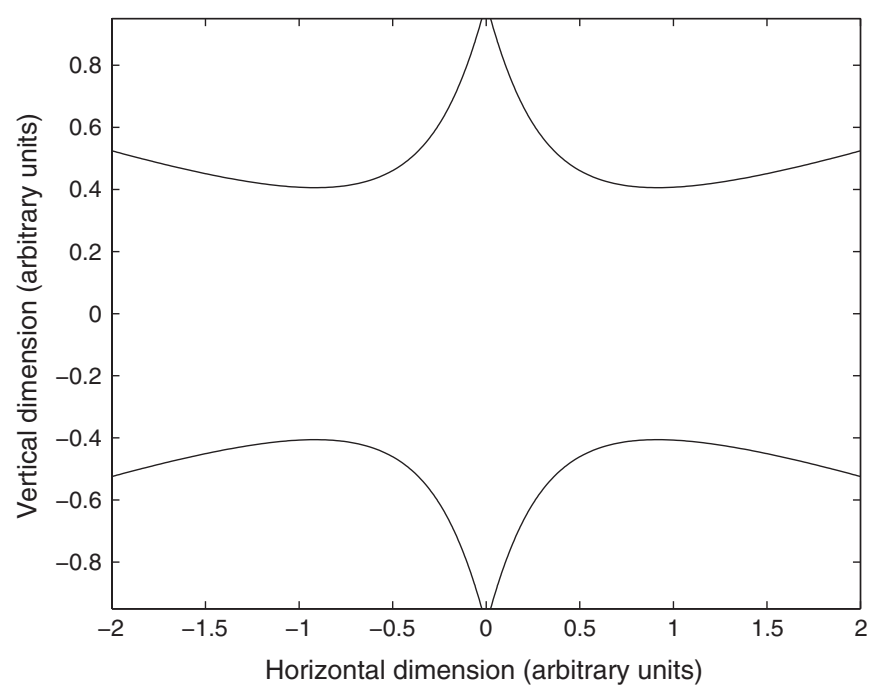

FIG. 6. Nonlinear lens magnet pole profile (arbitrary units).

lots of resonant islands. All these large resonances are absent in the integrable case. This comparison demonstrates the profit of using "integrable" lattices.

\section{NONLINEAR LENS}

The nonlinear kick (4) can be realized with two samecurrent wires in an iron-free lens [the position of the wires corresponds to the pole of the denominator in (4)], or with the conventional iron magnets. For example, if the kick in expression (5) were $F(z)=\frac{z}{z^{2}+1}$, the iron poles would look like ones shown in Fig. 6.

The field has the same signature as in a quadrupole case (the adjacent poles have opposite field), but unlike conventional quadrupoles, the poles lack 90 degrees symmetry. The pole profile is a smooth function of coordinates, much the same as in the case of quadrupole poles, where $y \propto \frac{1}{x}$, therefore the design of the nonlinear lens will be similar to the design of quadrupole (or other multipole) lenses.

\section{COMPARISON WITH OTHER APPROACHES}

Having described our approach, we would like to make a brief comparison with other methods to avoid chaos and increase the dynamic aperture of accelerator lattices. The vast amount of published material, aimed at practical results, can be divided into two groups.

The first group is focused on reducing the tune spread thus avoiding resonance crossing. The details of analytical procedures can be found in [12]; one example of eliminating the footprint for beam-beam effects can be found in [13]. The resulting systems with zero foot print are integrable- they can be obtained from linear systems by making an arbitrary canonical transformation of coordinates and momenta. The systems in these papers are drastically different in a sense that the goal of the present construction is to get as much betatron tune spread as possible, therefore the resonance crossings are unavoidable in principle.

The second group of papers, aimed at improved integrability, is focused on dynamic aperture limitations (see, e.g., $[14,15]$ ) and eliminating or controlling the resonance strengths (see, e.g., $[4,16]$ ). Papers $[4,5]$, mentioned in the Introduction, are very close in spirit to the goals of this paper-eliminating all resonances and achieving almost full integrability. This goal can be achieved (numerically, or analytically like in the present paper) in the 1D case. As it is clear from [5], the elimination of 2D resonances is a very tough procedure, taking into account the fact that the horizontal and vertical degrees of freedom are firmly coupled through Maxwell equations for the magnetic fields. The general situation is that if we want to have a large tune spread, the number of available knobs is not sufficient to create a quasi-integrable nonlinear 2D lattice. This paper suggests that the 2D coupling resonances can be efficiently suppressed by choosing the underlying linear lattice to have large beta function ratios at nonlinear lens position. Moreover, by introducing special lens profiles, like in the previous section, one can kill all 1D resonances, thus creating a realizable extremely nonlinear lattice with nearly regular motion.

We would like to remark that the integrability is a very fragile property and any nonlinear perturbation destroys it. For example, the thin lens presentation of the kick is an approximation and the difference between the real and thin lens transformations produces a perturbation. The perturbations can come from other sources, but in most cases, small perturbations cause relatively little change in the phase space (due to the so-called Kolmogorov-Arnol'dMoser theorem). This is always the case with real accelerators - the lattice design starts with an ideal linear lattice and then takes into account all other elements (sextupoles, octupoles, etc.), treating them as perturbations. In this paper, the ideal lattice, like in the linear case, has predictable motion with controllable or zero strength resonances, depending on parameters of the kick (4). But the tremendous difference with the standard linear lattice approach is that the nonlinear spread of betatron tunes is large from the very beginning and comparable with the betatron tune itself.

Concluding this section, we have to note that the goal of the approach, presented in the paper, is not to advance the theory of integrable systems but to make use of it in applications to accelerator problems. One example of such an application is the round colliding beam idea [1], where the practical conditions of creating the round beams were found. This was recently implemented with relative success [17]. We develop the approach here in the same vein. All the nonlinear kicks can be created by real 2D magnetic fields using lenses with special profiles. The linear lattices between them are a trivial piece that can be realized in many ways. The goal of such a lattice is to 
advance beam intensities and space charge limits, similar to the paper [7] idea of reducing beam halo by using nonlinear elements.

\section{CONCLUSION}

This paper demonstrates that there exist a variety of nonlinear 2D lattices with very large betatron tune spread and nearly integrable resonance-free motion with unlimited dynamic aperture. Possible lattice constructions and nonlinear kick realizations are discussed.

\section{ACKNOWLEDGMENTS}

The author thanks A. Aleksandrov and J. Holmes for useful comments and help in manuscript preparation. The research is sponsored by UT-Battelle, LLC, for the U.S. Department of Energy under Contract No. DE-AC0500OR22725.

[1] L. M. Barkov et al., Part. Accel. 31, 177 (1990).

[2] V. V. Danilov and E. A. Perevedentsev, CERN Report No. SL/Note 94-21(AP); CERN Report No. SL/Note 9474(AP).

[3] E. M. McMillan, Report No. UCRL-17795, University of California, Lawrence Radiation Laboratory, Berkeley, California, 1967; L. J. Laslett, E. M. McMillan, and J. Moser, Report No. NYO-1480-101, AEC Computing and Applied Mathematics Center, Courant Institute of Mathematical Sciences, New York University, 1968.

[4] C. C. Chow and J.R. Cary, Phys. Rev. Lett. 72, 1196 (1994).
[5] W. Wan and J. R. Cary, Phys. Rev. ST Accel. Beams 4, 084001 (2001).

[6] K. G. Sonnad and J. R. Cary, Phys. Rev. E 69, 056501 (2004).

[7] K. G. Sonnad and J. R. Cary, Phys. Rev. ST Accel. Beams 8, 064202 (2005).

[8] V. V. Danilov and E. A. Perevedentsev, in the Workshop on Beam-Beam Effects, Dubna, Russia, 1995; in Proceedings of the Particle Accelerators Conference, Vancouver, BC, Canada, 1997 (IEEE, New York, 1997).

[9] V. V. Danilov and V.D. Shiltsev, in Proceedings of the Particle Accelerator Conference, Vancouver, BC, Canada, 1997, Ref. [8].

[10] A. Otboev, Master's thesis, Novosibirsk University, Novosibirsk, Russia, 1997.

[11] V. V. Danilov et al., in Proceedings of the 18th Particle Accelerator Conference, New York, 1999 (IEEE, New York, 1999).

[12] H. Eliasson, in Nonlinear Dynamics, edited by G. Turchetti (World Scientific, Singapore, 1989).

[13] V. V. Danilov and V. D. Shiltsev, Report No. FERMILABFN-671, 1998.

[14] E. Todesco and M. Giovannozzi, Phys. Rev. E 53, 4067 (1996).

[15] W. Fischer, M. Giovannozzi, and F. Schmidt, Phys. Rev. E 55, 3507 (1997).

[16] M. Giovannozzi, D. Quatraro, and G. Turchetti, in Proceedings of the 11th European Particle Accelerator Conference, Genoa, 2008 (EPS-AG, Genoa, Italy, 2008).

[17] Yu. M. Shatunov, D. E. Berkaev, I. Koop, A. P. Lysenko, E. Perevedentsev, A. L. Romanov, P. Yu. Shatunov, D. B. Shwartz, and A. N. Skrinsky, in Proceedings of the 11th European Particle Accelerator Conference, Genoa, 2008, Ref. [16]. 